\title{
interview
}

\section{A picture of pressure}

\section{A sensor based on an array of piezoelectric $\mathrm{ZnO}$ nanowires on a light-emitting $\mathrm{GaN}$ substrate can generate high-resolution images of pressure distributions. Zhong Lin Wang tells Nature Photonics that it could lead to superior touch interfaces for electronic devices.}

How did you come up with the idea of creating the sensor?

I've been working on nanoscale piezoelectric effects for almost 10 years. In 2006, we published a paper in Science about a 'nanogenerator' that uses the piezoelectric effect to convert tiny mechanical movements into electricity. Later that year, we realized that this technology could lead to another application. If you produce a structure that consists of a semiconductor and a piezoelectric material, you can control the local charge distribution at the interface between the two materials by applying a strain in the structure. In effect, you can turn charge transport on and off by applying a strain. The piezoelectric polarization charge serves as a gate voltage, creating a straincontrolled transistor. The introduction of photon excitation gives rise to a related phenomenon, which we termed the piezophototronic effect in 2009. Our paper in this issue of Nature Photonics describes how we exploit this effect by using strain to alter the light emission from an array of nanowire light-emitting diodes (LEDs).

\section{How does this device work?}

Each nanowire LED acts as an independent pn junction, so that the local strain it experiences is indicated by the amount of light it emits. Light emission increases with increasing strain through the piezophototronic effect. Measuring the light emission from a high-density array of such LEDs allows the two-dimensional pressure distribution to be read out. The spatial resolution of these measurements is simply determined by the spacing between the nanowires. A big benefit is that measurements are performed in parallel using a single shot. We can generate single-shot maps very rapidly on a microsecond timescale. We presently use a camera to image the light emission at the base of the nanowires, but there's no reason why you couldn't use a bundle of optical fibres for readout at a remote location. You don't need to match one fibre to one nanowire; you could multiplex one fibre to several nanowires. We have managed to fabricate 22,000 nanowires in an area about the size of a fingertip, which allowed us to achieve an effective resolution of 6,300 dots per inch.

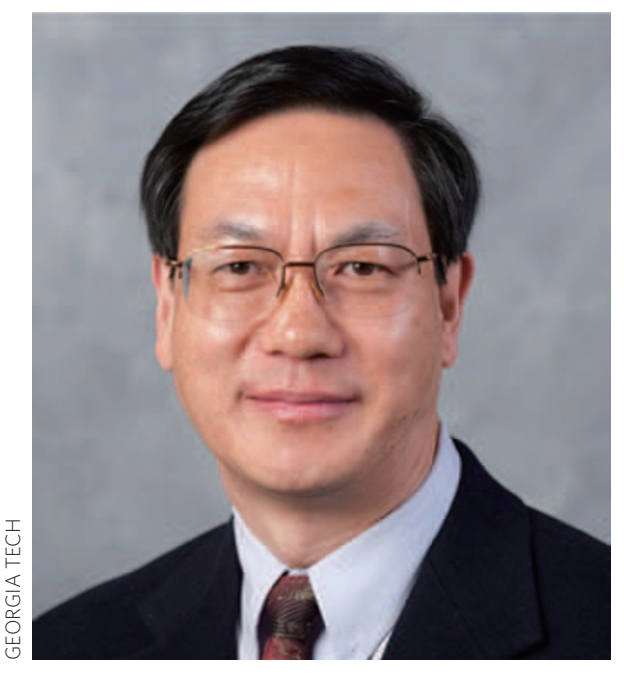

By combining a piezoelectric material and a lightemitting semiconductor, Zhong Lin Wang and co-workers have fabricated a sensor that lights up when strain is applied.

How do you make the sensors?

We usually use 2 inch $\times 2$ inch GaN wafers, but the beauty of the fabrication technique is that we grow the nanowires by a lowtemperature chemical method. The wafer is spin-coated with a polymer and then laser interference lithography is used to define the nanowire positions in a single shot. There is no bottleneck for large-scale fabrication, and we can easily scale to 4 -inch or 8-inch wafers.

\section{How robust is the sensor?}

Robustness is one reason why we use an array of nanowires rather than a large-area thin film of a piezoelectric material, which would crack after many deformations. The small size of the nanowires makes them very robust. You can elastically bend the zinc oxide nanowires $20^{\circ}$ off axis without breaking them. Dynamic vibration resonance tests reveal that a nanowire can survive $3.5 \times 10^{10}$ cycles before suffering from fatigue. In addition, using nanowires means that the effect remains localized, whereas the strain spreads across the film in a continuous thin film so that you lose the localization and spatial resolution.
What are its potential applications? In terms of applications, touchpads for human-machine interfaces are becoming extremely important. As both zinc oxide and indium tin oxide (which can be used as the electrode) are transparent, we can fabricate transparent nanowire arrays. This means that it could be put onto a mobile phone display without affecting the visualization of the display information. There are also security applications. For example, it could be used to very precisely read a fingerprint or a signature. In addition, dynamically sensing the local pressure permits a true electronic signature to be created by monitoring the speed and force used when writing the signature. For example, you may write your name quickly, whereas someone trying to forge your signature may write it much more slowly. Another very important application is the development of artificial or smart skin, which mimics the sense of touch in fingers for use in robotics.

\section{What are your future plans for} this technology?

We are presently looking at making devices on flexible substrates. Because the growth temperature is only $85^{\circ} \mathrm{C}$, flexible substrates made from polymers can be used. We are also considering making a self-powered design that uses nanogenerators to power the nanowire LEDs, with all the components integrated into a single package. We are also assessing the materials we use in order to optimize performance and improve the dynamic range. The time resolution is currently $1 \mathrm{~ms}$, but the piezoelectric effect and optical emission processes are almost instantaneous with nanosecond or faster responses. The packaging materials lower the temporal response. By optimizing the packaging materials, a microsecond time response should be achievable. Another point is how to actually use this technology with devices such as mobile phones. We really want to transfer this technology to industry.

\section{INTERVIEW BY OLIVER GRAYDON}

Zhong Lin Wang and co-workers have an Article on their nanowire pressure sensor on page 752 of this issue. 